\title{
Experimental Results and Modeling Advances in the Study of the Nanoparticle Field Extraction Thruster AIAA-2007-5254
}

\author{
Louis Musinski ${ }^{*}$, Thomas Liu ${ }^{\dagger}$, Brian Gilchrist ${ }^{\ddagger}$, Alec Gallimore ${ }^{\S}$, and Michael Keidar ${ }^{* *}$ \\ University of Michigan, Ann Arbor, MI, 48109
}

\begin{abstract}
This paper presents experimental results and modeling advances concerning the nanoparticle Field Extraction Thruster (nanoFET) concept under development at the University of Michigan. The nanoFET concept offers an electric propulsion approach that can have a highly adjustable charge-to-mass ratio and electrostatic acceleration that potentially could span a specific impulse range from $\sim 100 \mathrm{~s}$ to $\sim 10,000 \mathrm{~s}$ and thrust power ranging from microwatts to many tens of kilowatts at high efficiency. Here, we model the extraction process for both spherical and cylindrical particles from an insulating liquid and compare the threshold extraction electric field with the minimum electric field required for the liquid to become unstable. The model suggests that there exists a range of usable particles that can be extracted prior to the onset of liquid instability. Experimental data collected appears to follow the trend predicted by the extraction model.
\end{abstract}

\section{Nomenclature}

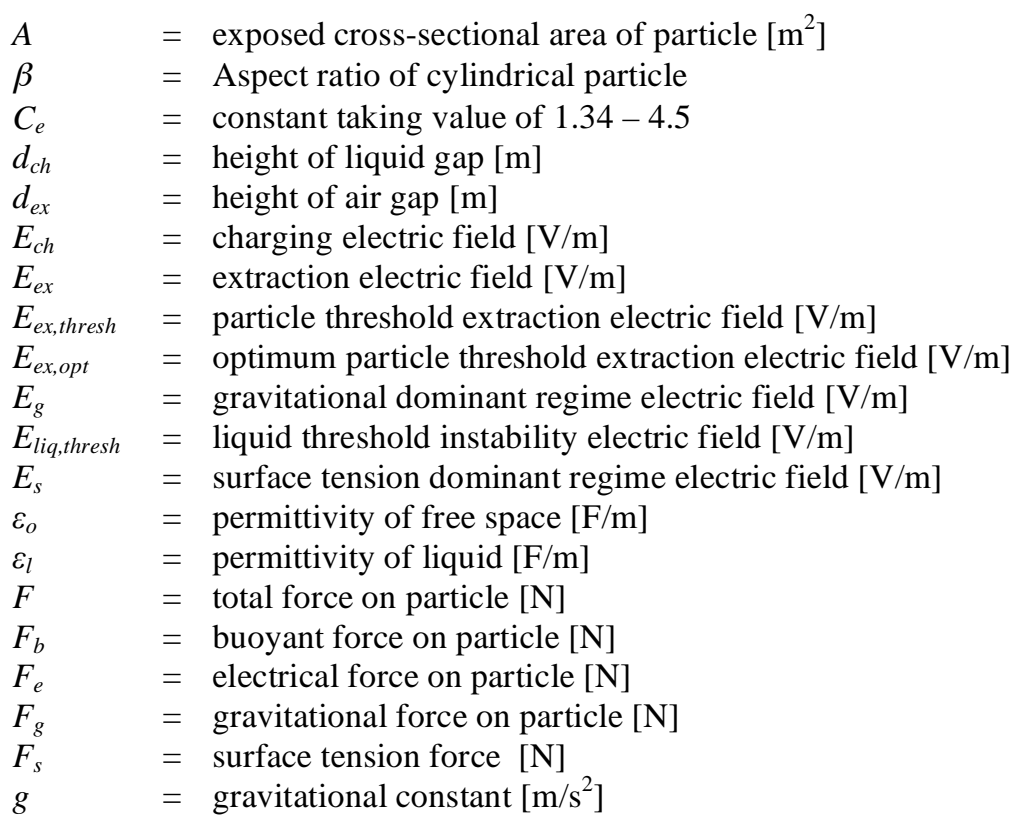

\footnotetext{
* Graduate Student, Electrical Engineering, louisdm@umich.edu, AIAA Student Member

${ }^{\dagger}$ Graduate Student, Aerospace Engineering, liutm@umich.edu, AIAA Student Member

¥ Professor, Electrical Engineering \& Space Systems, gilchrst@ umich.edu, AIAA Associate Fellow

${ }^{\S}$ Arthur F. Thurnau Professor, Aerospace Engineering \& Applied Physics, rasta@umich.edu, AIAA Associate Fellow

** Assistant Research Professor, Aerospace Engineering, keidar@umich.edu, Senior AIAA Member
} 


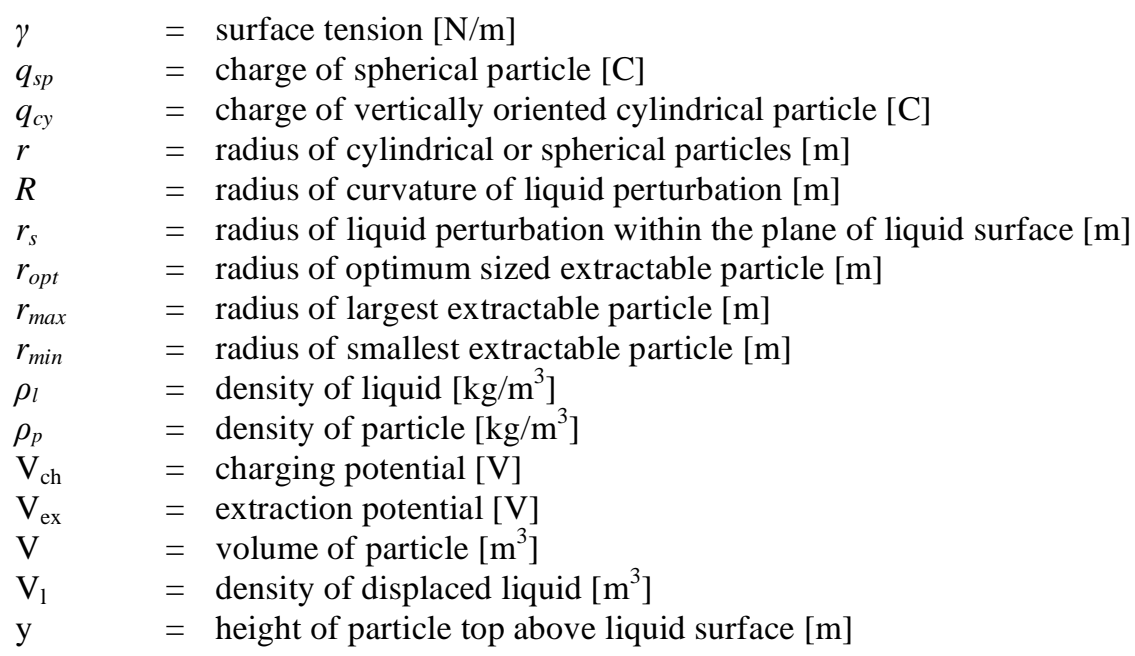




\section{Introduction}

$\mathrm{T}$ The nanoparticle field extraction thruster, termed nanoFET, is under development at the University of Michigan. This new electrostatic thruster technology appears feasible using nanoparticles as propellant and utilizing micro- and nano-electromechanical systems (NEMS/MEMS) to transport, charge, extract, and accelerate the nanoparticles. ${ }^{1}$

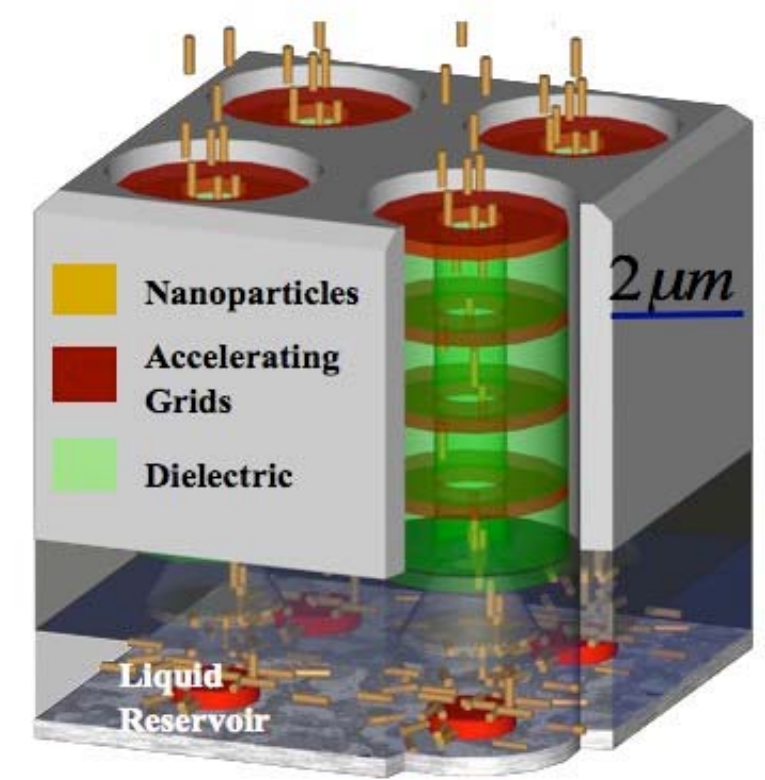

Figure 1: Schematic showing four nanoparticle emitters.

Figure 1 is a schematic of four nanoparticle emitters used in the MEMS/NEMS based nanoFET thruster concept where a multi-layer grid establishes the critical electric fields to charge, extract, accelerate, and eject conducting nanoparticles (one example shown in Figure 2 ) $^{2}$ from the surface of an insulating liquid used to transport these particles. These nanoparticles will likely have characteristic sizes ranging from $1 \mathrm{~nm}$ to over $100 \mathrm{~nm}$.

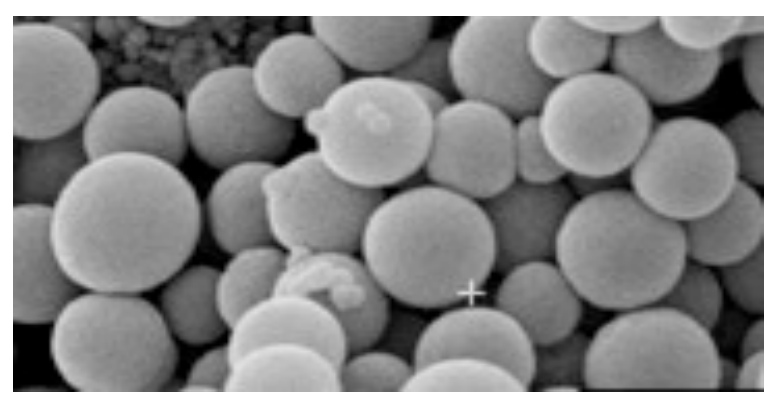

Figure 2: Example of a collection of unsorted nanoparticles in the $10 \mathrm{~nm}$ diameter range.

To understand how nanoFET works, Figure 3 depicts a model of a single emission zone from the nanoparticle thruster using an insulating liquid. The nanoparticles are initially housed in a low vapor pressure liquid-filled storage reservoir. These nano-spheres or cylinders would then be transported through a microfluidic transport system to emission zones. These zones are comprised of multiple layers of stacked electrodes with millions of micron-sized channels for particle acceleration. Beneath the accelerating channels are electrical charging pads that are submersed in the low vapor pressure liquid. The layers of stacked electrodes and the charging pads are electrically biased to produce strong electric fields in the accelerating channels and within the liquid reservoir.

Particles delivered to the emission zones through the microfluidic transport system become electrically charged when they contact one of the charging pads. After acquiring sufficient charge, the 
particles are transported by the electric field to the liquid surface, extracted through the surface, accelerated in the channels, and finally ejected to produce thrust

It is also possible to consider a similar configuration, but using a slightly conducting liquid where nanoparticles arrive at the liquid surface and are preferentially charged relative to the surrounding surface, thus resulting in extraction.

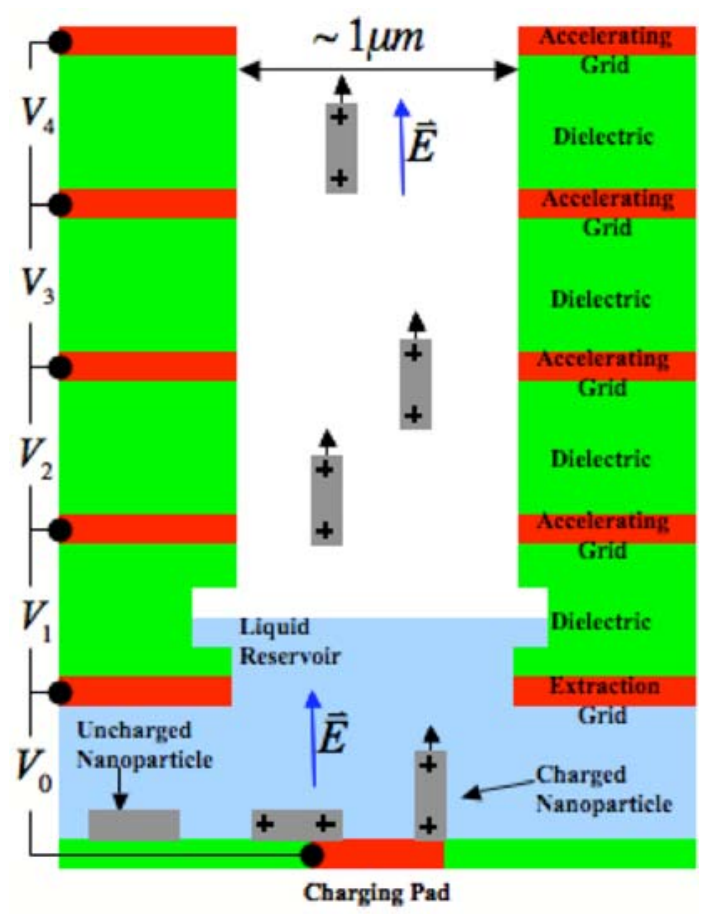

Figure 3: Cross section of a single emission channel used in the microparticle emitter prototype.

Using nanoparticles as opposed to ions or charged liquid droplets may provide many operational advantages, including the following: ${ }^{3,4}$

- Enormous specific impulse range at high thrust efficiency: The ability to tune propulsion characteristics via different nanoparticle dimensions and charge states has the potential of permitting thrust efficiencies over $85 \%$ for a specific impulse range of $100 \mathrm{~s}$ to $10,000 \mathrm{~s}$. Such high efficiencies could result in thrust-to-power ratios, especially at low specific impulse, that are orders of magnitude greater than stateof-the-art ion and Hall thrusters. Consequently, a nanoFET thruster could operate at high specific impulse in cruise mode and yet switch to a high thrust and low Isp mode when needed. This flexibility provides a wider margin for mission designers to accommodate off-nominal mission scenarios as well as dynamic retasking of space assets to take advantage of in-flight opportunities.

For example, by using just three types of nanoparticles, the nanoFET system should span an approximate Isp range from $150 \mathrm{~s}$ to $10,000 \mathrm{~s}$. Three possible carbon nanotube particles are listed in Table 1.

\begin{tabular}{ccc}
\hline \hline Diameter $[\mathrm{nm}]$ & Length $[\mu \mathrm{m}]$ & Isp range $[\mathrm{s}]$ \\
\hline 16 & 3 & $150-750$ \\
4 & 3 & $750-2,700$ \\
1 & 3 & $2,700-10,000$ \\
\hline \hline
\end{tabular}

Table 1: Carbon nanotube particles enabling nanoFET to span 150-10,000 second range.

Note that the Isp range could potentially be covered with over $85 \%$ thrust efficiency using just the three particle types as shown in Figure $4 .^{5}$ Such performance can be obtained at reasonable acceleration voltages between 500 to $10,000 \mathrm{~V}$ due to the MEMS gated structures that provide the extraction and acceleration electric fields. Note that in the nanoFET system, efficiency losses may be due to viscous drag in the liquid, charge loss to the liquid, particle impingement on the gates, and beam defocusing. 


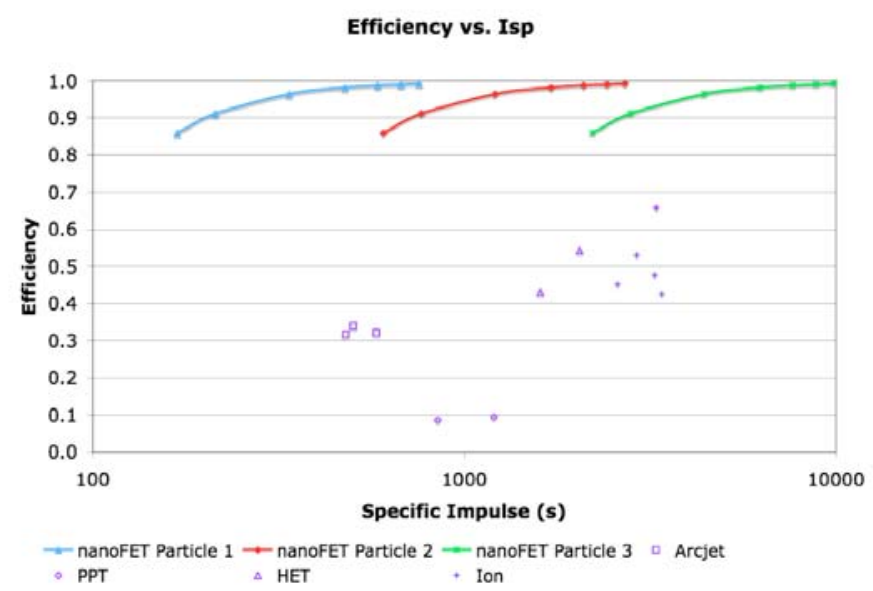

Figure 4: Possible efficiency performance of nanoFET for specific impulse range compared with other electric propulsion systems. The nanoparticles are specified in Table 1.

NanoFET's thrust-to-power performance compared to other state-of-the-art thruster types is shown in Figure 5. This level of performance could provide mission designers with the flexibility to engage a high thrust mode to climb out of gravity wells, perform abort scenarios or emergency maneuvers, and reduce trip times.

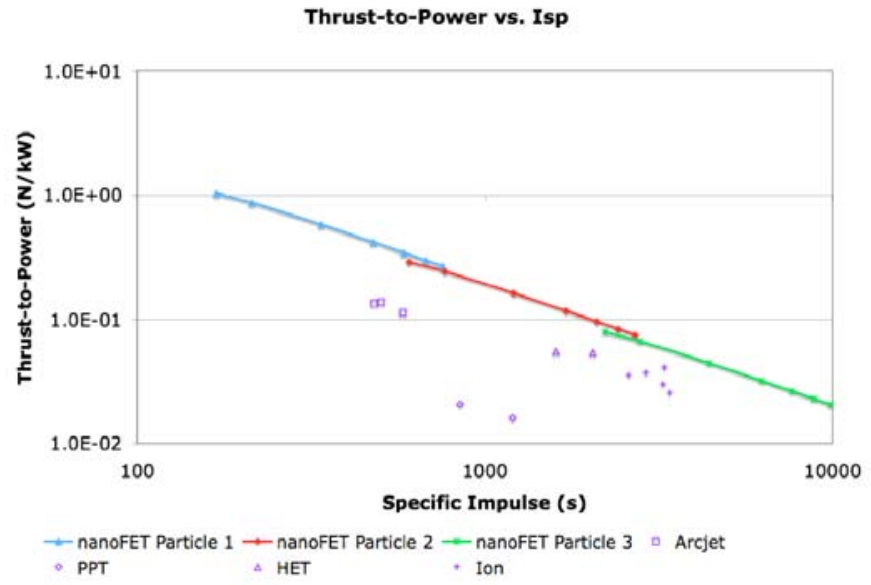

Figure 5: High thrust-to-power ratio for nanoFET compared to other electric propulsion systems. The nanoparticles are specified in Table 1.

- Highly integrated system: The use of MEMS technology enables a "flat panel" thruster design that incorporates power processing as well as nanoparticle manufacture, storage, feed, extraction, and acceleration. Such compact design simplifies propulsion system integration and lowers thruster specific mass. Because different regions of nanoFET can emit particles of opposite polarity, neutralizer requirements are simplified as nanoFET is a self-neutralizing thruster.

- Geometrically scalable: A "flat panel" design allows the nanoFET array size to be scalable with power, thus permitting a single ground qualified engine type to be used in applications ranging from nanosatellites operating at a few watts to space tugs or space stations in the hundreds of kilowatts range. Such "plug-and-play" functionality provides greater spacecraft design flexibility and significant cost savings.

- Longer operational lifetime: Charging of the nanoparticles is accomplished without ionization, meaning greater reliability and the absence of cathodes and charge exchange collisions that are the principal lifetime limiters of current electric propulsion systems. Propellant charging, as opposed to propellant ionizing, also accounts for part of the efficiency gains nanoFET affords. 


\section{Particle Extraction: Modeling and Experimental Results}

Using high strength electric fields to extract millimeter sized charged particles from an insulating liquid has previously been demonstrated, ${ }^{6}$ but it is imperative that we show particle extraction is still possible as the particle size is reduced down to the micron and even further down to the nano-scale. This section discusses models developed for spherical and cylindrical particle extraction from an insulating liquid along with experimental results and their implications.

It is important to understand that the models presented in this section make assumptions in order to simplify and understand the process of particle extraction and how it scales with particle size. Without many of these assumptions, the problem could not be solved statically, and a time dependant simulation would be required. We would expect a time dependant simulation to provide more accurate results, but can be very time consuming because as the particle size is scaled down to below the micron range, the time step required becomes very small. Therefore, using a time dependant simulation to explore the extraction of micro- and nanoparticles is not currently the best approach. The models presented in this section will sacrifice the accuracy of the time dependant simulation in order to determine how the extraction process changes with particle size and is intended to show the trends of particle extraction as the particle size changes.

The following are assumptions used with the derivations for particle extraction from an insulating liquid that may hurt the performance of the models.

- The particle arrives at the liquid surface with Negligible kinetic energy:

The models use the assumption that the particle arrives at the liquid surface with zero kinetic energy, which may have several consequences. In most foreseeable cases the particle will arrive at the liquid surface with some kinetic energy since it will be traversing though the liquid with a non-zero velocity. The velocity of the particle in the liquid will be dependant on many different factors including the particle's charge, size, and shape, along with the applied electric field and the liquid's viscosity.

One consequence of ignoring the particle's velocity when it arrives at the liquid surface is the model will not account for the kinetic energy that the liquid surface will absorb while slowing the particle. This will lead to overestimating the electric field required for particle extraction and will be more important for large and fast particles since the kinetic energy of the particle is proportional to the product of the mass and the square of the velocity. An analysis of particle velocity within the liquid showed that the kinetic energy that the liquid surface will absorb while slowing the particle is negligible for particles less than several tens of microns in size. As the particle size increases, the energy can potentially become significant.

A second consequence of ignoring the particle's velocity is that the model will not account for the liquid draining from the particle's surface. The models assume that the liquid will always drain in such a manner that a negligibly and uniformly thin layer of liquid will coat exposed surfaces of the particle. But, this will not always be the case. We expect that if the particle extraction time is fast compared to the draining time of the liquid, then the liquid surface coating the particle will be thicker at the top of the particle than at its sides. Conversely, if the particle is moving slowly compared to the drain time of the liquid, the liquid surface coating the particle will be thicker at the sides of the particle than at its top.

The significance of this effect is that the effective mass of the extracted particle includes both the particle's mass as well as the mass of the liquid pulled along. This will lead to underestimating the electric field required for particle extraction and will be more important for smaller and faster particles. It is expected to be more important for small particles because the mass of the additional liquid will be a larger percentage of the effective mass, and it is expected be more important for fast particles because the liquid will have less time to drain and will then be extracted with the particle.

- The liquid surface is charge free:

The models assume that the liquid surface remains uncharged during particle extraction. But, even though the various liquids used for particle extraction are very good insulators, it is known that when an electric field is applied to the liquids, charge will slowly accumulate on their surfaces. For the liquids in consideration, the accumulation of charge is very slow and takes on the order of minutes for the surface to become charged.

As charge accumulates slowly on the liquid's surface, the electric field within the liquid will begin to decrease which means that the amount of charge that the particle will acquire from 
the bottom electrode will decrease. In addition, if a charged particle is brought to a charged liquid surface, there will be less field focusing on the particle itself. This implies that overtime it may become more difficult to extract particles and this will lead to underestimating the electric field required for particle extraction for all particles.

But, we know from previous experiments with silicone oil that its charging time constant is on the order of several minutes. Therefore, as long as particle extraction experiments can be performed on the order of seconds, this assumption is valid.

Although it is possible that these assumptions may hurt the performance of the models, we expect the models to provide a reasonable trend as the particle size is varied, and to provide a reasonable range of particle sizes that can be extracted under desirable conditions.

\section{Spherical Particle Extraction with Submersed Charging Grid}

The setup that will be used for particle extraction is shown in Figure 6 and includes a three-grid system with a hole cut out of the center grid to allow passage of the particle. The lower electrode gap will be completely filled with the insulating liquid and the upper gap will be air, vacuum, or another gas. Two high voltage power supplies will be used to generate strong electric fields in both electrodes gaps. The electric field in the lower gap, $\mathrm{E}_{\mathrm{ch}}$, will be referred to as the charging electric field and the field in the upper gap, $\mathrm{E}_{\mathrm{ex}}$, will be referred to as the extraction electric field.

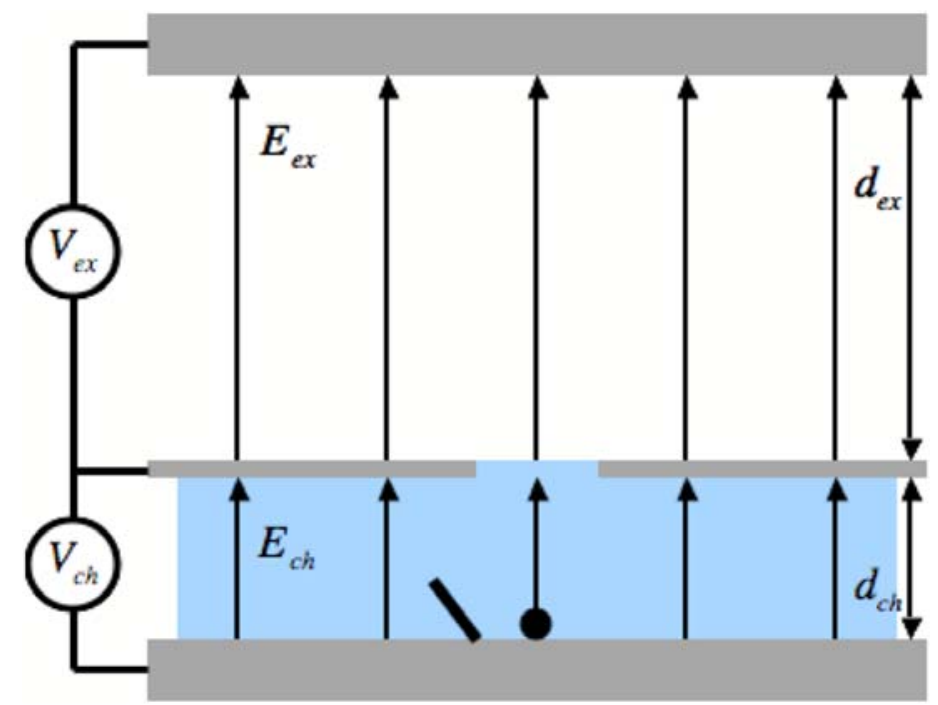

Figure 6: Setup for particle extraction from insulating liquid.

The use of two power supplies as opposed to just one decouples the two electric fields, which can be approximated as

$$
E_{e x}=\frac{V_{e x}}{d_{e x}} \quad \text { (1) } \quad \text { and } \quad E_{c h}=\frac{V_{c h}}{d_{c h}}
$$

as long as the diameter of the hole in the middle grid is less than or equal to both electrode gaps, $d_{\mathrm{ex}}$ and $\mathrm{d}_{\mathrm{ch}}$.

A spherical particle in contact with the bottom electrode will become electrostatically charged as

$$
q_{\mathrm{sp}}=\frac{2 \pi^{3}}{3} r^{2} \varepsilon_{\ell} E_{c h}
$$

according Félici. ${ }^{7}$ Once charged, the particle is transported to the liquid surface by the electric force acting on it. For this model, we will assume that the transport time is much less than the charging time constant of 
the liquid. Therefore, the particle will not lose significant charge to the liquid during transport and will arrive at the liquid surface fully charged.

Once arriving at the liquid surface, four forces will act on the charged particle as shown in Figure 7. These forces are (1) the electrical force attempting to pull the particle from the liquid, (2) the gravitational force pulling the particle down, (3) the buoyant force pushing the particle up, and (4) the surface tension force resisting the extraction of the particle. The following subsections will briefly discuss these four forces as well as provide an expression for each. An important assumption is that a negligibly and uniformly thin layer of liquid will cover the exposed surfaces of the particle.

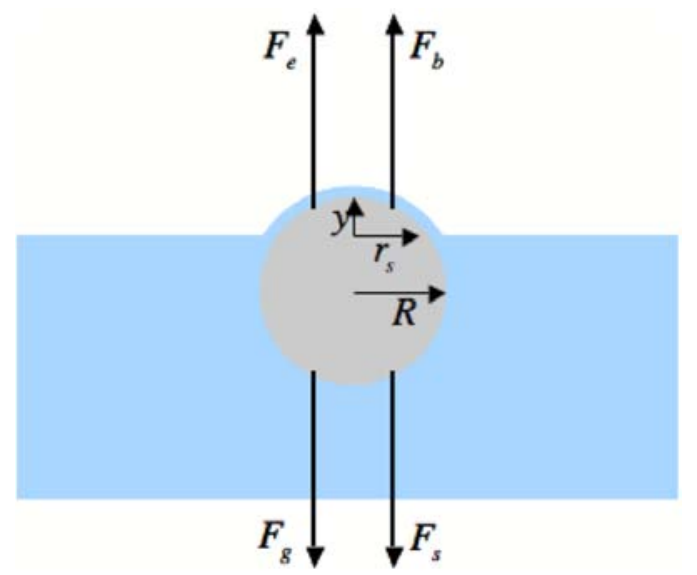

Figure 7: Forces on spherical particle at the liquid surface during extraction.

- Electrical Force

The electrical force acting on the particle is a result of the extraction electric field acting on the total charge on the particle and is given as

$$
F_{e}=q_{s p} E_{e x}=\frac{2 \pi^{3}}{3} r^{2} \varepsilon_{\ell} E_{c h} E_{e x}
$$

\section{- Surface Tension Force}

The surface tension force can be modeled as

$$
F_{s}=\left(\frac{2 \gamma}{R}\right) A
$$

where $\gamma$ is the surface tension of the liquid, $R$ is the radius of curvature of the liquid perturbation, and $\mathrm{A}$ is the exposed cross-sectional area of the particle. $\mathrm{R}$ can be rewritten in terms of the distance that the particle top protrudes above the plane of the liquid surface, $y$, and the radius of the perturbation within the plane of the liquid surface, $r_{s}$.

$$
R=\frac{r_{s}^{2}+y^{2}}{2 y}
$$

The surface tension force can then be written as

$$
F_{s}=\left(\frac{4 \gamma y}{r_{s}^{2}+y^{2}}\right) A
$$

- Gravitational Force 
The gravitational force acting on the particle is simply

$$
F_{g}=\rho_{p} V g
$$

where $\rho_{\mathrm{p}}$ is the density of the particle, $\mathrm{V}$ is the volume of the particle, and $\mathrm{g}$ is the gravitational constant.

- Buoyant Force

The buoyant force is the upward force on the particle due to the volume of liquid it is displacing and is expressed as

$$
F_{b}=\rho_{\ell} V_{\ell} g \quad(9),
$$

were $\rho_{\ell}$ is the density of the liquid and $\mathrm{V}_{\ell}$ is the volume of liquid displaced by the particle.

To find the threshold extraction electric field for particle extraction, the forces acting on the particle are summed and set equal to zero.

$$
\begin{gathered}
F=F_{e}+F_{b}-F_{g}-F_{s}=0 \\
F=\frac{2 \pi^{3}}{3} r^{2} \varepsilon_{\ell} E_{c h} E_{e x}+g\left(V_{\ell} \rho_{\ell}-V \rho_{p}\right)-\left(\frac{4 \gamma y}{r_{s}^{2}+y^{2}}\right) A=0
\end{gathered}
$$

The minimum extraction electric field can be solved for and expressed as

$$
E_{e x}=\left[\left(\frac{4 A y \gamma}{r_{s}^{2}+y^{2}}\right)+g\left(V \rho_{p}-V_{\ell} \rho_{\ell}\right)\right]\left[\frac{3}{2 \pi^{3} r^{2} \varepsilon_{\ell} E_{c h}}\right]
$$

We have chosen to determine the cross-sectional exposed area of the particle when the center of the particle is even with the plane of the liquid surface so that half of the sphere is exposed.

$$
A=\pi r^{2}(13) \quad r_{s}=r(14) \quad y=r(15)
$$

This position was chosen because it maximizes the surface tension force and if the particle can be extracted from this position, it can be extracted from any other position. We also know that the volume of the particle and the volume of the displaced liquid can be expressed as

$$
V=\frac{4}{3} \pi r^{3}(16) \quad \text { and } \quad V_{\ell}=\frac{2}{3} \pi r^{3} \text { (17), respectively. }
$$

After substituting back in, the threshold extraction electric field can be expressed as

$$
E_{\text {ex,thresh }}=\frac{3 \gamma+g r^{2}\left(2 \rho_{p}-\rho_{\ell}\right)}{r \pi^{2} \varepsilon_{\ell} E_{c h}}
$$

It is important to understand that the threshold extraction electric field can be broken down into two terms: the surface tension dominant term, $\mathrm{E}_{\mathrm{s}}$, and the gravitational dominant term, $\mathrm{E}_{\mathrm{g}}$. 


$$
E_{s}=\frac{1}{r}\left(\frac{3 \gamma}{\pi^{2} \varepsilon_{\ell} E_{c h}}\right)
$$

$$
E_{g}=r\left(\frac{g\left(2 \rho_{p}-\rho_{\ell}\right)}{\pi^{2} \varepsilon_{\ell} E_{c h}}\right)
$$

Note that the surface tension dominant term scales inversely with the particle size while the gravitational term scales directly with the particle size. This implies that for very small particles the threshold electric field required for particle extraction will increase as the particle size is reduced, but for very large particles the threshold electric field will increase as particle size is increased. Therefore, there should be a particle size that lies between the very small and very large particles that can be extracted with the minimum threshold electric field. To find the optimum particle size that can be extracted with the lowest extraction electric field and its corresponding electric field, the derivative of the threshold extraction electric field with respect to $r$ is set equal to zero and solved for $r$.

$$
E_{e x, o p t}=\frac{2 \sqrt{3 \gamma g\left(2 \rho_{p}-\rho_{\ell}\right)}}{\pi^{2} \varepsilon_{\ell} E_{c h}}
$$

$$
r_{o p t}=\sqrt{\frac{3 \gamma}{g\left(2 \rho_{p}-\rho_{\ell}\right)}}
$$

Recall that when a high strength electric field is applied to a liquid surface, it is possible for the liquid surface to become unstable and to form cone like structures called Taylor cones, which may emit charged liquid droplets referred to as electrospray. To determine if it is possible for particles to be extracted from a liquid using electric fields lower than required for the liquid surface to become unstable, we will compare the threshold extraction electric field with the minimum electric field required to initiate an instability in a liquid surface. We will use a model for liquid instability that we developed which follows Tonks's ${ }^{8}$ model fairly closely. The minimum electric field applied to an unperturbed liquid surface required to initiate an instability in the surface was determined to be

$$
E_{\text {liq,thresh }}=C_{e}^{1 / 4}\left(\frac{\gamma \rho_{\ell} g}{\varepsilon_{o}^{2}}\right)^{1 / 4}
$$

where $\mathrm{C}_{\mathrm{e}}$ is a constant whose value lies between 1.34 and 4.5.

Since the threshold particle extraction electric field scales with the inverse of $r$ when in the surface tension dominant regime and scales with $r$ when in the gravitational dominant regime with a minimum in the middle, it is possible for there to exist a range of particle sizes where extraction occurs with electric fields lower than required for the liquid to become unstable. The predicted maximum sized particle that can be extracted prior to the liquid becoming unstable is

$$
\begin{gathered}
r_{\max }=\frac{\pi^{2} \varepsilon_{\ell} E_{c h}\left(\frac{C_{e} \gamma g \rho_{\ell}}{\varepsilon_{o}^{2}}\right)^{1 / 4}+\sqrt{\pi^{4} \varepsilon_{\ell}^{2} E_{c h}^{2}\left(\frac{C_{e} \gamma g \rho_{\ell}}{\varepsilon_{o}^{2}}\right)^{1 / 2}-12 g \gamma\left(2 \rho_{p}-\rho_{\ell}\right)}}{2 g\left(2 \rho_{p}-\rho_{\ell}\right)} \\
r_{\max } \approx \frac{\pi^{2} \varepsilon_{\ell} E_{c h}\left(\frac{C_{e} \gamma g \rho_{\ell}}{\varepsilon_{o}^{2}}\right)^{1 / 4}}{g\left(2 \rho_{p}-\rho_{\ell}\right)}
\end{gathered}
$$

Similarly, the predicted minimum sized particle that can be extracted prior to the liquid becoming unstable is 


$$
\begin{aligned}
r_{\min }=\frac{\pi^{2} \varepsilon_{\ell} E_{c h}\left(\frac{C_{e} \gamma g \rho_{\ell}}{\varepsilon_{o}^{2}}\right)^{1 / 4}}{-\sqrt{\pi^{4} \varepsilon_{\ell}^{2} E_{c h}^{2}\left(\frac{C_{e} \gamma g \rho_{\ell}}{\varepsilon_{o}^{2}}\right)^{1 / 2}-12 g \gamma\left(2 \rho_{p}-\rho_{\ell}\right)}} \\
r_{\min } \approx \frac{3 \gamma\left(2 \rho_{p}-\rho_{\ell}\right)}{\pi^{2} \varepsilon_{\ell} E_{c h}\left(\frac{C_{e} \gamma g \rho_{\ell}}{\varepsilon_{o}^{2}}\right)^{1 / 4}}
\end{aligned}
$$

Figure 8 shows a plot of the expected threshold extraction and threshold instability electric fields when extracting spherical particles from $100 \mathrm{cSt}$ silicone using a particle charging electric field of 10 $\mathrm{MV} / \mathrm{m}$. The silicone oil has a surface tension of $0.0209 \mathrm{~N} / \mathrm{m}$, a density of $965 \mathrm{~kg} / \mathrm{m}^{3}$, and a relative dielectric constant of 1.97. We chose to set the constant $\mathrm{C}_{\mathrm{e}}$ equal to 4.5 and used particles made from aluminum $\left(2700 \mathrm{~kg} / \mathrm{m}^{3}\right)$.

Spherical Particle Extraction (Al) from 100 cSt Silicone Oil Charging Field $=10 \mathrm{MV} / \mathrm{m}$

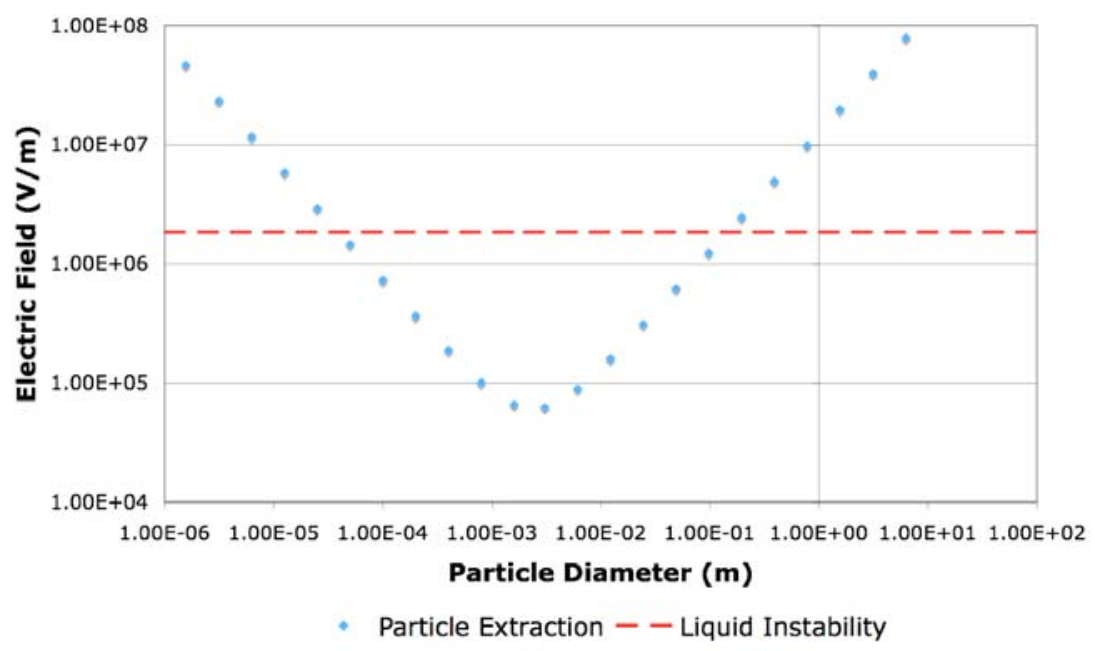

Figure 8: Plot of the expected threshold extraction and threshold instability electric fields when extracting aluminum spherical particles from $100 \mathrm{cSt}$ silicone oil using a particle charging electric field of $10 \mathrm{MV} / \mathrm{m}$.

The plot in Figure 8 shows that there exists a range of spherical particle sizes, which should be extractable from $100 \mathrm{cSt}$ silicone oil prior to the onset of the liquid instability. As expected the extraction electric fields increase for small particles as the particle size is reduced because the surface tension becomes more dominant. Also, the extraction electric fields increase for large particles as the particle size is increased because the gravitational force becomes more dominant. Table 2 lists the expected minimum, maximum, and optimum particle sizes that can be extracted from the $100 \mathrm{cSt}$ silicone oil when using a charging field of $10 \mathrm{MV} / \mathrm{m}$ for various materials.

\begin{tabular}{|c|c|c|c|}
\hline Material & $\begin{array}{c}\text { Minimum Extractable } \\
\text { Particle Diameter }(\mathrm{m})\end{array}$ & $\begin{array}{c}\text { Optimum Extractable } \\
\text { Particle Diameter }(\mathrm{m})\end{array}$ & $\begin{array}{c}\text { Maximum Extractable } \\
\text { Particle Diameter }(\mathrm{m})\end{array}$ \\
\hline Aluminum & $3.97 * 10^{-5}$ & $2.40^{-5} 10^{-3}$ & $1.45^{-3} 10^{-1}$ \\
\hline Indium & $3.97 * 10^{-5}$ & $1.37 * 10^{-3}$ & $4.72^{-3} 10^{-2}$ \\
\hline
\end{tabular}

Table 2: List of the minimum, maximum, and optimum particle sizes that can be extracted from the $100 \mathrm{cSt}$ silicone oil when using a charging field of $10 \mathrm{MV} / \mathrm{m}$ 
The table shows that we can expect the smallest spherical particle capable of being extracted at electric fields lower than required to initiate a liquid instability from $100 \mathrm{cSt}$ silicone oil to be on the order of $40 \mu \mathrm{m}$. This particle size will change very little with the density of the particle because it is the surface tension that limits particle extraction. On the other hand, the maximum and optimum particle sizes for extraction do change significantly with the particle density since gravity is the limiting force. Therefore, low-density particles can be extracted with larger diameters than high-density particles.

Since the surface tension dominant regime of the particle extraction electric field scales directly with the surface tension of the liquid and the liquid instability threshold field scales with the surface tension to the one-fourth power, we expect to be able to extract smaller particles as the surface tension is reduced. Figure 9 shows a plot of the minimum, maximum, and optimum particle sizes that can be extracted from an insulating liquid as a function of surface tension when using a charging field of $10 \mathrm{MV} / \mathrm{m}$ to extract aluminum spheres.

Spherical Particle Extraction Range (AI) Charging Field $=10 \mathrm{MV} / \mathrm{m}$

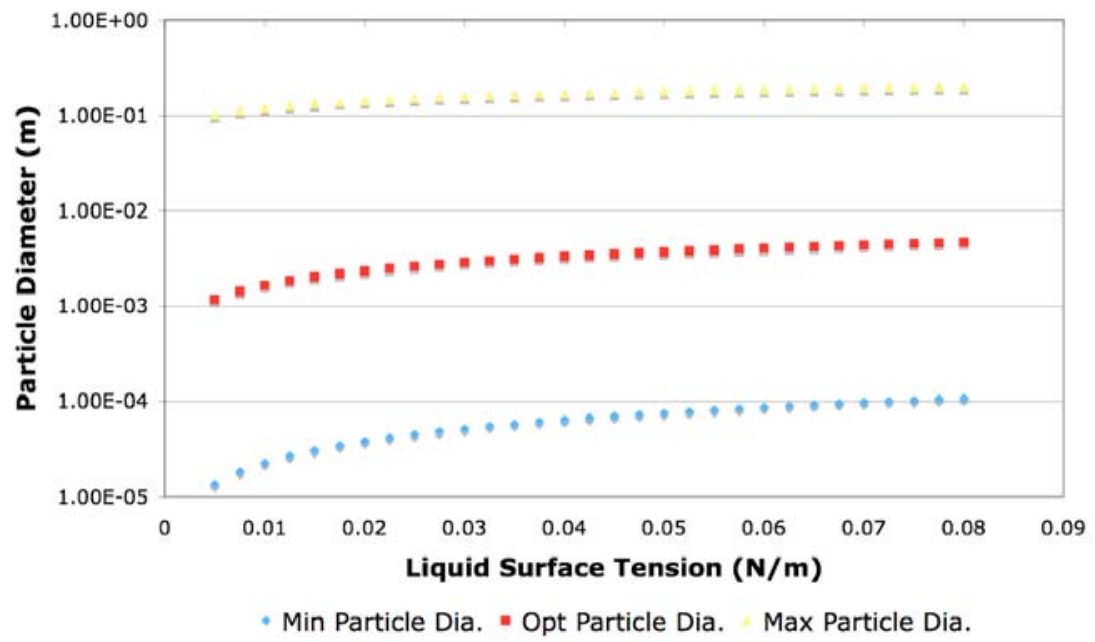

Figure 9: Plot of the minimum, maximum, and optimum particle sizes that can be extracted from an insulating liquid as a function of surface tension when using a charging field of $10 \mathrm{MV} / \mathrm{m}$ to extract aluminum spheres.

As expected, smaller particles can be extracted prior to the onset of the liquid instability as the surface tension of the liquid is reduced. But, according to the plot, extractable particle sizes cannot be reduced below tens of microns for reasonable liquid surface tensions. As it is, silicone oil has a very low surface tension $(\sim 0.0209 \mathrm{~N} / \mathrm{m})^{9}$ compared to most other liquids. It may be possible to reduce the surface tension of silicone oil by using surfactants, but at this point it is unclear what effects they may have on other properties of the liquid.

This model suggests that the smallest spherical particles that can be extracted from $100 \mathrm{cSt}$ silicone oil when using a charging electric field of $10 \mathrm{MV} / \mathrm{m}$ is on the order of $40 \mu \mathrm{m}$, which is much larger than the particle size required to obtain reasonable charge-to-mass ratios for operation as a thruster. This does not come as a surprise, and it has always been expected that cylindrical particles would be needed to achieve the desired exhaust velocities, which will be discussed in a later section.

\section{Spherical Particle Extraction without Submersed Charging Grid}

The previous section discussed the model used for particle extraction when a submersed charging grid was used to decouple the charging and the extraction electric fields. But, since our current experimental setup does not implement a submersed charging grid, we have not yet been able to experimentally test this model. This section modifies the particle extraction model when there is not a submersed charging grid as shown in Figure 10, and then compares it to experimental results. 


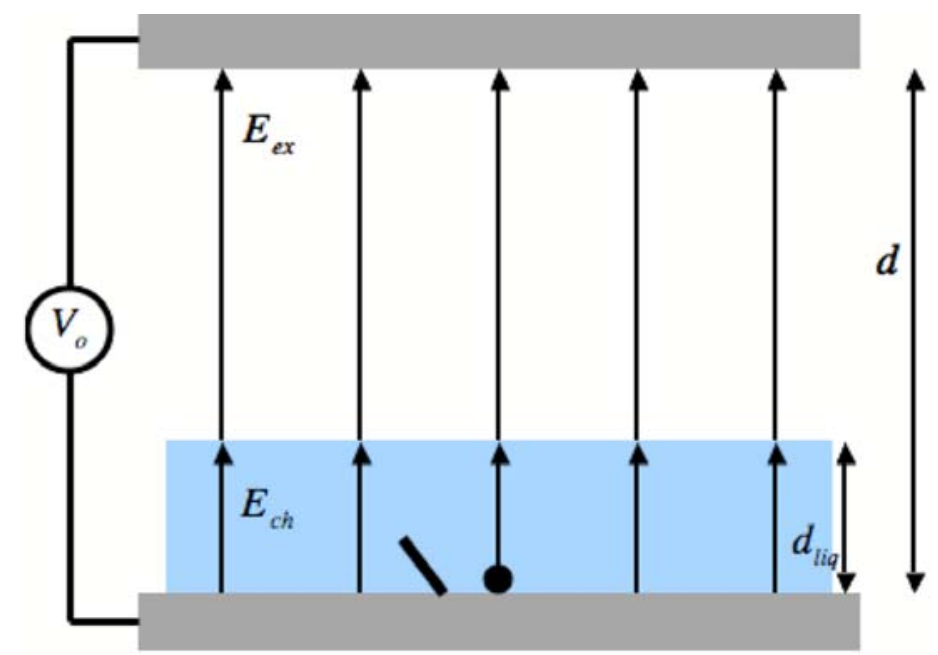

Figure 10: Setup for particle extraction from insulating liquid without submersed charging grid.

Since only one power supply is used to generate both the charging and extraction electric fields, they are dependant on one another and the relationship between the two can e expressed as

$$
\varepsilon_{o} E_{e x}=\varepsilon_{\ell} E_{c h}
$$

when there is no free charge at the liquid/air boundary. The charging electric field can be solved for and substituted back into the expression derived previously for the threshold extraction electric field to find the new threshold electric field for particle extraction when the submersed charging grid is eliminated.

$$
E_{e x, t h r e s h}=\sqrt{\frac{3 \gamma+g r^{2}\left(2 \rho_{p}-\rho_{\ell}\right)}{r \pi^{2} \varepsilon_{o}}}
$$

Again, it is important to note that there is a surface tension dominant term and a gravitational dominant term. The surface tension dominant term scales inversely with the square root of the particle size while the gravitational term scales directly with the square root of the particle size. Recall from Equation 18 that the surface tension and gravitational dominant terms scale inversely and directly with the particle size, respectively, when using a submersed charging grid.

As before, we can calculate the optimum, maximum, and minimum particles sizes that can be extracted prior to the onset of the liquid instability.

$$
r_{o p t}=\sqrt{\frac{3 \gamma}{g\left(2 \rho_{p}-\rho_{\ell}\right)}} \quad \text { (30) } \quad r_{\max } \approx \frac{\pi^{2} \sqrt{C_{e} \gamma g \rho_{\ell}}}{g\left(2 \rho_{p}-\rho_{\ell}\right)} \quad \text { (31) } \quad r_{\min } \approx \frac{3 \gamma}{\pi^{2} \sqrt{C_{e} \gamma g \rho_{\ell}}}
$$

Table 3 lists the minimum, maximum, and optimum particle sizes that can be extracted from the $100 \mathrm{cSt}$ silicone oil without using a submersed charging grid for various materials.

\begin{tabular}{|c|c|c|c|}
\hline Material & $\begin{array}{c}\text { Minimum } \\
\text { Extractable } \\
\text { Particle } \\
\text { Diameter }(\mathrm{m})\end{array}$ & $\begin{array}{c}\text { Optimum } \\
\text { Extractable } \\
\text { Particle } \\
\text { Diameter }(\mathrm{m})\end{array}$ & $\begin{array}{c}\text { Maximum } \\
\text { Extractable } \\
\text { Particle } \\
\text { Diameter }(\mathrm{m})\end{array}$ \\
\hline Aluminum & $4.40^{-4} 10^{-4}$ & $2.40^{*} 10^{-3}$ & $1.31 * 10^{-2}$ \\
\hline Indium & $4.78 * 10^{-3}$ & $1.37 * 10^{-3}$ & $3.92 * 10^{-3}$ \\
\hline
\end{tabular}

Table 3: List of the minimum, maximum, and optimum particle sizes that can be extracted from the $100 \mathrm{cSt}$ silicone oil without using a submersed charging grid. 
The table shows that we can expect the smallest spherical particle capable of being extracted at electric fields lower than required to initiate a liquid instability from $100 \mathrm{cSt}$ silicone oil to be on the order of several hundred microns. The table also shows that when a submersed charging grid is not used, the range of extractable particles is much narrower.

To test the spherical particle extraction model, we measured the minimum applied electric field required to extract various sized aluminum spheres from $100 \mathrm{cSt}$ silicone oil. Unfortunately, the range of particle sizes that we could test was very limited. When exploring the surface tension dominant regime, the particles had to be larger than many hundred microns so that they could be extracted prior to the onset of the liquid instability. The gravitational dominant regime could not be explored because the minimum electric field required to lift the particle from the bottom electrode was higher than the threshold extraction electric field. Both of these factors limiting the testing range will be improved once our new prototype, which uses a submersed charging grid, is finished.

Figure 11 shows a plot of the expected threshold extraction and threshold instability electric fields when extracting spherical particles from $100 \mathrm{cSt}$ silicone when not using a submersed charging grid along with experimental measurements.

Spherical Particle Extraction (AI) from 100 cSt Silicone Oil No Charging Grid

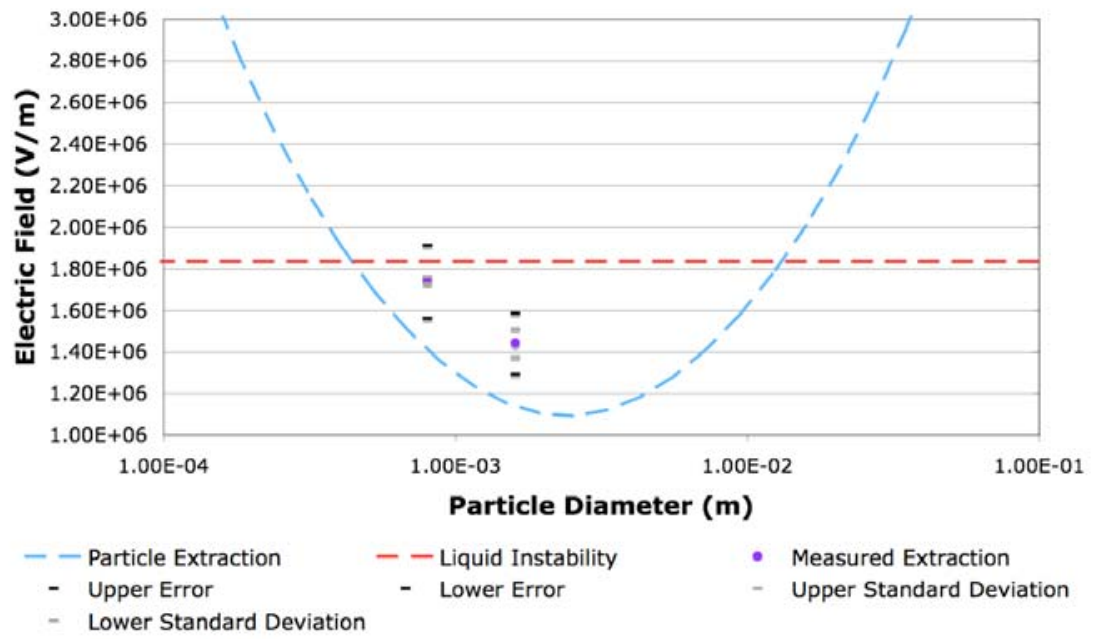

Figure 11: Plot of the expected threshold extraction and threshold instability electric fields when extracting aluminum spherical particles from $100 \mathrm{cSt}$ silicone oil using along with experimental results.

The plot in Figure 11 shows that there exists a range of spherical particle sizes that can be extracted from $100 \mathrm{cSt}$ silicone oil prior to the onset of the liquid instability. As expected, the extraction electric fields increase for small particles as the particle size is reduced because the surface tension becomes more dominant. Also, the extraction electric fields increase for large particles as the particle size is increased because the gravitational force becomes more dominant. The two experimental data points indicate that the model under predicts the threshold particle extraction electric field. This is most likely because the extracted particles are carrying additional liquid mass along, which would make particle extraction more difficult as discussed earlier. But, the measured data does agree with the expected trend in the surface tension dominant regime.

\section{Cylindrical Particle Extraction with Submersed Charging Grid}

The model for cylindrical particle extraction follows the model for spherical particle extraction very closely, and uses the same setup shown in Figure 6. The only significant difference between the models is the electrical force used to extract particles through the liquid surface, which arises because the differently shaped particles are charged differently. According to Félici, a vertically oriented cylindrical particle in contact with the bottom electrode will become charged as 


$$
q_{\mathrm{cy}}=\pi \frac{l^{2}}{\ln \left(\frac{2 l}{r}\right)-1} \varepsilon_{\ell} E_{c h}
$$

The resulting electric force used to extract the particle through the liquid surface is

$$
F_{e}=q_{c y} E_{c h}=\pi \frac{l^{2}}{\ln \left(\frac{2 l}{r}\right)-1} \varepsilon_{\ell} E_{c h} E_{e x}
$$

Using the same gravitational, buoyant, and surface tension forces as in the spherical particle extraction model but tailored specifically for cylindrical particles, the threshold extraction electric field can be expressed as

$$
E_{\text {ex,thresh }}=\frac{\left[2 \gamma+2 g r^{2} \beta\left\{\rho_{p}-\rho_{\ell}\left(1-\frac{1}{2 \beta}\right)\right\}\right][\ln (4 \beta)-1]}{4 r \beta^{2} \varepsilon_{\ell} E_{c h}}
$$

where $\beta$ is the aspect ratio of the particle, which is defined as the ratio of the particle length to the particle diameter. Just as with the spherical particle model, the threshold extraction electric field can be broken down into a surface tension dominant term which scales inversely with the particle radius and a gravitational dominant term, which scales directly with the particle radius.

$$
E_{s}=\frac{1}{r} \frac{\gamma[\ln (4 \beta)-1]}{2 \beta^{2} \varepsilon_{\ell} E_{c h}}(36) \quad E_{g}=r \frac{\left[g\left\{\rho_{p}-\rho_{\ell}\left(1-\frac{1}{2 \beta}\right)\right\}\right][\ln (4 \beta)-1]}{2 \beta \varepsilon_{\ell} E_{c h}}
$$

As before, we can calculate the optimum, maximum, and minimum particles sizes that can be extracted prior to the onset of the liquid instability.

$$
\begin{gathered}
r_{o p t}=\sqrt{\frac{2 \gamma}{g\left(2 \beta \rho_{p}-(2 \beta-1) \rho_{\ell}\right)}} \\
r_{\max } \approx \frac{4 \beta^{2} \varepsilon_{\ell} E_{c h}\left(\frac{C_{e} \gamma g \rho_{\ell}}{\varepsilon_{o}^{2}}\right)^{1 / 4}}{g\left(2 \beta \rho_{p}-(2 \beta-1) \rho_{\ell}\right)[\ln (4 \beta)-1]} \\
r_{\min } \approx \frac{\gamma[\ln (4 \beta)-1]}{2 \beta^{2} \varepsilon_{\ell} E_{c h}\left(\frac{C_{e} \gamma g \rho_{\ell}}{\varepsilon_{o}^{2}}\right)^{1 / 4}}
\end{gathered}
$$

Figure 12 shows a plot of the expected threshold extraction and threshold instability electric fields when extracting cylindrical particles from $100 \mathrm{cSt}$ silicone using a particle charging electric field of 10 $\mathrm{MV} / \mathrm{m}$. We set the constant $\mathrm{C}_{\mathrm{e}}$ equal to 4.5 , and used aluminum particles. 
Cylindrical Particle Extraction (Al) from 100 cSt Silicone Oil Charging Field $=10 \mathrm{MV} / \mathrm{m}$

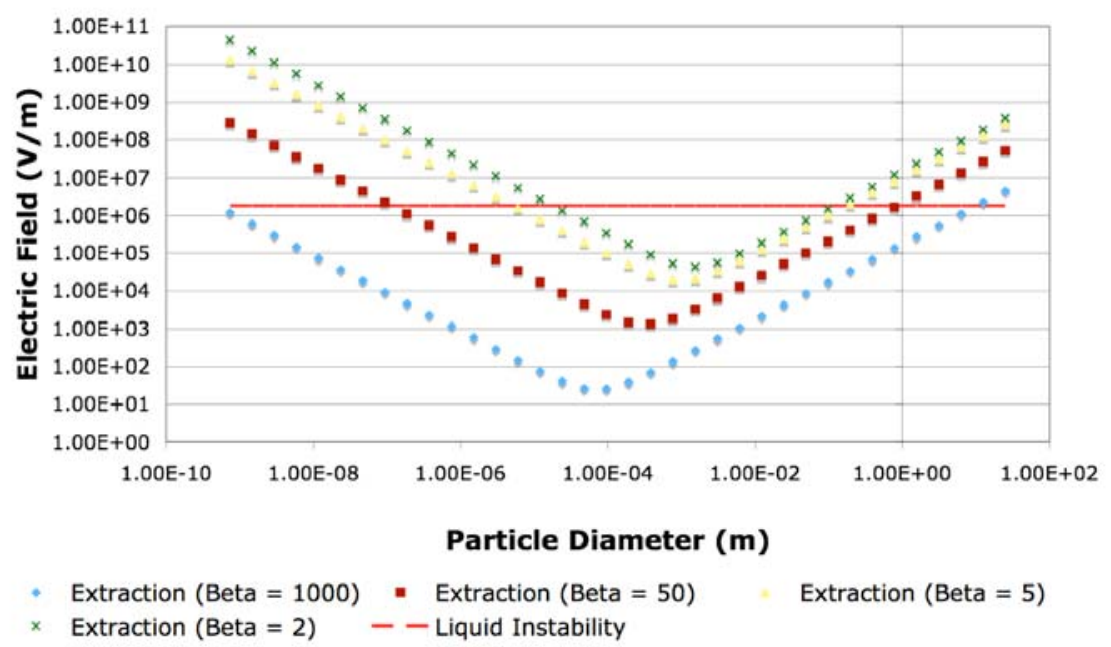

Figure 12: Plot of the expected threshold extraction and threshold instability electric fields when extracting aluminum cylindrical particles from $100 \mathrm{cSt}$ silicone using a particle charging electric field of $10 \mathrm{MV} / \mathrm{m}$.

The plot in Figure 12 shows that there exists a range of cylindrical particle sizes that can be extracted from $100 \mathrm{cSt}$ silicone oil prior to the onset of the liquid instability. As expected the extraction electric fields increase for particles with small radii as the particle radius is reduced because the surface tension dominates. Also, the extraction electric fields increase for particles with large radii as the particle radius is increased because the gravitational force dominates. In addition, increasing the particle's aspect ratio reduces the required extraction electric field in both the gravitational and surface tension dominant regimes, providing a much larger range of extractable particles. Table 4 lists the minimum, maximum, and optimum particle sizes that can be extracted from the $100 \mathrm{cSt}$ silicone oil when using a charging field of 10 $\mathrm{MV} / \mathrm{m}$ for various materials.

\begin{tabular}{|c|c|c|c|c|}
\hline Material & Aspect Ratio & $\begin{array}{c}\text { Minimum } \\
\text { Extractable } \\
\text { Particle } \\
\text { Diameter }(\mathrm{m})\end{array}$ & $\begin{array}{c}\text { Optimum } \\
\text { Extractable } \\
\text { Particle } \\
\text { Diameter }(\mathrm{m})\end{array}$ & $\begin{array}{c}\text { Maximum } \\
\text { Extractable } \\
\text { Particle } \\
\text { Diameter }(\mathrm{m})\end{array}$ \\
\hline Aluminum & 1000 & $4.76^{*} 10^{-10}$ & $7.01 * 10^{-5}$ & 10.3 \\
\hline Tungsten & 1000 & $4.76^{*} 10^{-10}$ & $2.16^{*} 10^{-5}$ & $9.80^{*} 10^{-1}$ \\
\hline Aluminum & 50 & $1.12^{*} 10^{-7}$ & $3.13 * 10^{-4}$ & $8.71 * 10^{-1}$ \\
\hline Tungsten & 50 & $1.12^{*} 10^{-7}$ & $9.66^{*} 10^{-5}$ & $8.31^{-2} 10^{-2}$ \\
\hline
\end{tabular}

Table 4: List of the minimum, maximum, and optimum cylindrical particle diameters that can be extracted from the $100 \mathrm{cSt}$ silicone oil when using a charging field of $10 \mathrm{MV} / \mathrm{m}$

The table shows that we can expect the smallest cylindrical particles capable of being extracted at electric fields lower than required to initiate a liquid instability from $100 \mathrm{cSt}$ silicone oil to be on the order of $1 \mathrm{~nm}$ when an aspect ratio of 1000 is used and on the order of $110 \mathrm{~nm}$ when an aspect ratio of 50 is used. Particle sizes on this scale are ideal for use as nanoFET propellant because they will provide the desired charge-to-mass ratios. Note that the three particle sizes listed in Table 1 that could be used to span an Isp range from $150 \mathrm{~s}$ up to $10,000 \mathrm{~s}$ should be extractable prior to the onset of liquid instability according to this model.

\section{Cylindrical Particle Extraction without Submersed Charging Grid}

Just as with the spherical particle extraction model, we cannot verify the cylindrical particle model using our current experimental setup because it does not implement a submersed charging grid. Therefore, 
we will have to modify the model when there is not a submersed charging grid as shown in Figure 10. Using the boundary condition in Equation 28, the charging electric field can be expressed in terms of the extraction electric field, and the threshold electric field required for cylindrical particle extraction without a submersed charging grid can be expressed as

$$
E_{\text {ex,thresh }}=\sqrt{\frac{\left[\gamma+g r^{2} \beta\left\{\rho_{p}-\rho_{\ell}\left(1-\frac{1}{2 \beta}\right)\right\}[\ln (4 \beta)-1]\right.}{2 r \beta^{2} \varepsilon_{o}}}
$$

Again, there is a surface tension dominant term and a gravitational dominant term, which scale inversely with the square root of the particle radius, and directly with the square root of the particle radius, respectively. The optimum, maximum, and minimum particles sizes that can be extracted prior to the onset of the liquid instability are

$$
\begin{gathered}
r_{o p t}=\sqrt{\frac{2 \gamma}{g\left(2 \beta \rho_{p}-(2 \beta-1) \rho_{\ell}\right)}}(42), \\
r_{\max }=\frac{2 \beta^{2} \varepsilon_{o}\left(\frac{C_{e} \gamma g \rho_{\ell}}{\varepsilon_{o}^{2}}\right)^{1 / 2}+\sqrt{4 \beta^{4} \varepsilon_{o}^{2}\left(\frac{C_{e} \gamma g \rho_{\ell}}{\varepsilon_{o}^{2}}\right)-2 g \gamma\left(2 \beta \rho_{p}-(2 \beta-1) \rho_{\ell}\right)[\ln (4 \beta)-1]^{2}}}{g\left(2 \beta \rho_{p}-(2 \beta-1) \rho_{\ell}\right)[\ln (4 \beta)-1]} \text { and } \\
r_{\min }=\frac{2 \beta^{2} \varepsilon_{o}\left(\frac{C_{e} \gamma g \rho_{\ell}}{\varepsilon_{o}^{2}}\right)^{1 / 2}-\sqrt{4 \beta^{4} \varepsilon_{o}^{2}\left(\frac{C_{e} \gamma g \rho_{\ell}}{\varepsilon_{o}^{2}}\right)-2 g \gamma\left(2 \beta \rho_{p}-(2 \beta-1) \rho_{\ell}\right)[\ln (4 \beta)-1]^{2}}}{g\left(2 \beta \rho_{p}-(2 \beta-1) \rho_{\ell}\right)[\ln (4 \beta)-1]}
\end{gathered}
$$

Table 5 lists the minimum, maximum, and optimum particle sizes that can be extracted from the $100 \mathrm{cSt}$ silicone oil without using a submersed charging grid for various materials.

\begin{tabular}{|c|c|c|c|c|}
\hline Material & Aspect Ratio & $\begin{array}{c}\text { Minimum } \\
\text { Extractable } \\
\text { Particle } \\
\text { Diameter }(\mathrm{m})\end{array}$ & $\begin{array}{c}\text { Optimum } \\
\text { Extractable } \\
\text { Particle } \\
\text { Diameter }(\mathrm{m})\end{array}$ & $\begin{array}{c}\text { Maximum } \\
\text { Extractable } \\
\text { Particle } \\
\text { Diameter }(\mathrm{m})\end{array}$ \\
\hline Aluminum & 1000 & $5.11^{-9} 10^{-9}$ & $7.01 * 10^{-5}$ & $9.62 * 10^{-1}$ \\
\hline Tungsten & 1000 & $5.11^{-5} 10^{-9}$ & $2.16^{*} 10^{-5}$ & $9.13 * 10^{-2}$ \\
\hline Aluminum & 50 & $1.20 * 10^{-6}$ & $3.13^{*} 10^{-4}$ & $8.12^{-4} 10^{-2}$ \\
\hline Tungsten & 50 & $1.21 * 10^{-6}$ & $9.66^{*} 10^{-4}$ & $7.74 * 10^{-3}$ \\
\hline
\end{tabular}

Table 5: List of the minimum, maximum, and optimum particle sizes that can be extracted from the $100 \mathrm{cSt}$ silicone oil without using a submersed charging grid.

The table shows that we can expect the smallest cylindrical particles capable of being extracted at electric fields lower than required to initiate a liquid instability from $100 \mathrm{cSt}$ silicone oil to be on the order of $5 \mathrm{~nm}$ when an aspect ratio of 1000 is used and on the order of 1 micron when an aspect ratio of 50 is used. We also see that when a submersed charging grid is not used, the range of extractable particles becomes much narrower. 
To test this extraction model, we measured the minimum applied electric field required to extract various sized aluminum and tungsten cylinders from $100 \mathrm{cSt}$ silicone oil. Figure 13 shows plots of the expected threshold extraction and threshold instability electric fields when extracting cylindrical particles from $100 \mathrm{cSt}$ silicone when not using a submersed charging grid along with experimental measurements. Plot (a) shows how the threshold extraction field varies with the particle aspect ratio and plot (b) shows how the threshold extraction field varies with the particle diameter.

(a)
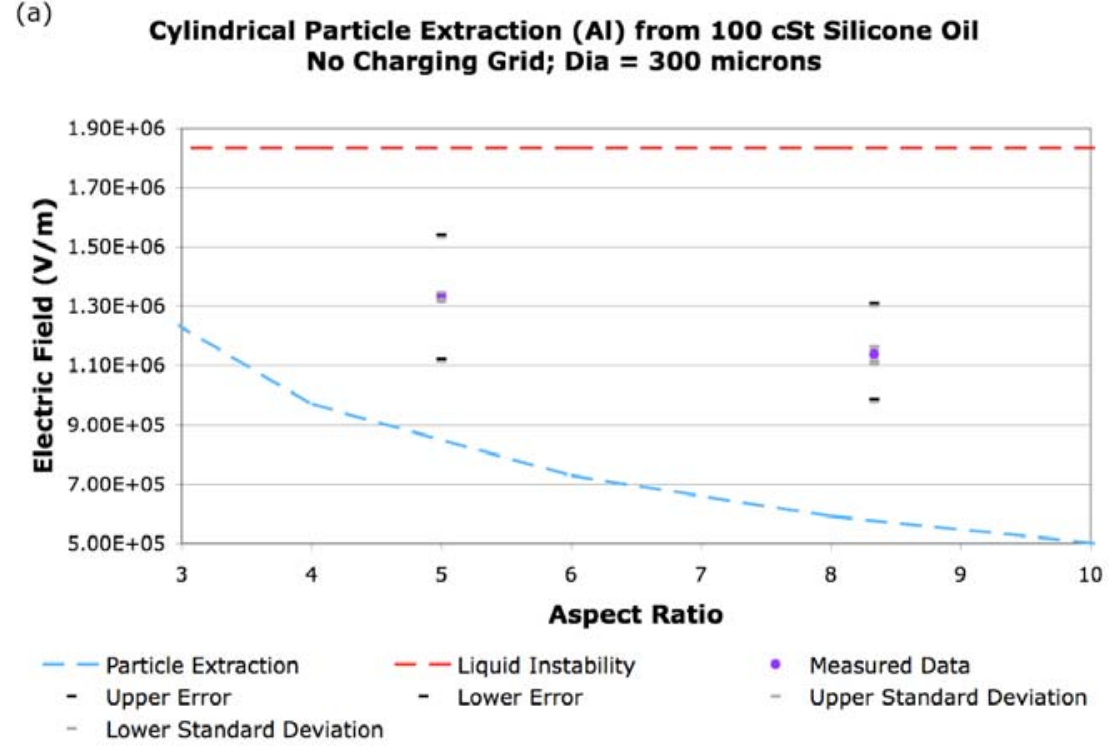

(b)

Cylindrical Particle Extraction (W) from 100 cSt Silicone Oil No Charging Grid; Beta $=15$

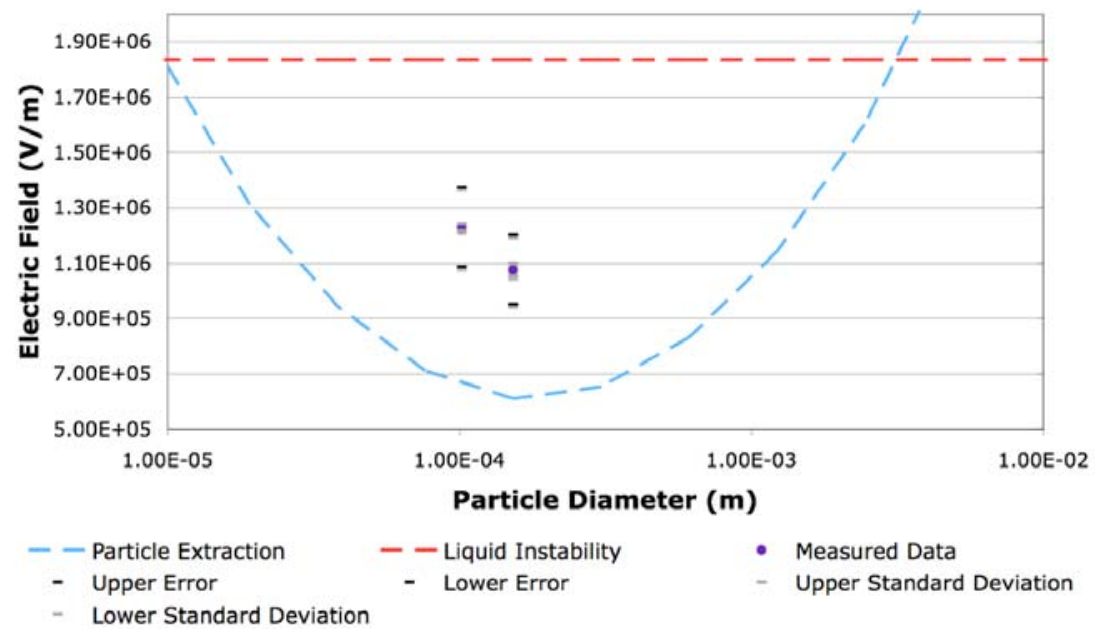

Figure 13: Plots of the expected threshold extraction and threshold instability electric fields when extracting aluminum and tungsten cylindrical particles from $100 \mathrm{cSt}$ silicone oil along with experimental results.

The plots in Figure 13 show that there exists a range of cylindrical particle sizes that can be extracted from $100 \mathrm{cSt}$ silicone oil prior to the onset of the liquid instability. As expected, the extraction electric fields increase for small radii particles as the radius is reduced because the surface tension becomes more dominant. Also, the extraction electric fields increase for large radii particles as the particle radius is increased because the gravitational force becomes more dominant. In addition, the extraction electric field decreases as the aspect ratio is increased. The experimental data indicates that the model under predicts the threshold particle extraction electric field, which is most likely because the extracted particles are carrying additional liquid mass just as with the spherical particles. But, the measured data does agree with the 
expected trend in the surface tension dominant regime.

\section{Conclusion}

We have modeled the extraction process for both spherical and cylindrical particles from an insulating liquid and compared the threshold extraction electric field with the minimum electric field required for the liquid to become unstable. The models suggest that there exists a range of particles that can be extracted prior to the onset of liquid instability, where small particles are limited by the surface tension and large particles are limited by gravity. Reducing the liquid's surface tension should allow smaller particles to be extracted. Experimental data collected in the surface tension dominant regime appears to follow the trend predicted by the extraction models.

According the cylindrical particle extraction model, the high aspect ratio cylinders desired for use with nanoFET should be extractable prior to the onset of the liquid instability.

1 L. Musinski, T. Liu, B. Gilchrist, A. Gallimore, M. Keidar. "Scalable Flat-Panel Nano-Particle MEMS/NEMS Thruster.” International Electric Propulsion Conference (IEPC), October 31 - November 4, 2005.

2 Behan, Niall, "Nanomedicin and Drug delivery at the University of Limerick," The University of Limerick. http://www.ul.ie/elements/Issue4/behan.htm

3 T. Liu, M. Keidar, L. Musinski, A. Gallimore, B. Gilchrist. "Theoretical Apects of Nanoparticle Electric Propulsion." 42nd AIAA/ASME/SAE/ASEE Joint Propulsion Conference and Exhibit, Sacramento, CA, AIAA2006-4335, July 9-12, 2006

4 T. Liu, L. Musinski, P. Patel, A. Gallimore, B. Gilchrist, M. Keidar. "Nanoparticle Electric Propulsion for Space Exploration." STAIF-2007, Albuquerque, NM, February 11-15, 2007

5 Wertz, Larson, "Space Mission Analysis and Design $3^{\text {rd }}$ Ed." 1999.

6 L. Musinski, T. Liu, B. Gilchrist, A. Gallimore, M. Keidar. "Nanoparticle Electric Propulsion: Experimental Results." 42nd AIAA/ASME/SAE/ASEE Joint Propulsion Conference and Exhibit, Sacramento, CA, AIAA-20064803, July 9-12, 2006

${ }^{7}$ Felici, N. Rev. Gen. Elect., 75, pp. 1145-1160, 1966

8 Tonks, L., "A Theory of Liquid Surface Rupture by a Uniform Electric Field," Physical Review, Vol. 48, 15 September 1935, pp. 562-8.

9 "Clearco Pure Silicone Fluids: Product Information." Clearco Products Co., Inc. Bensalem, PA 19020 